\title{
Lack of an association between the XRCC1 Arg399GIn polymorphism and gastric cancer based on a meta-analysis
}

\author{
B.M. Liu ${ }^{1,2 *}$, T.M. Liu ${ }^{3 *}$, B.S. You ${ }^{4}$ H.Y. You ${ }^{2}$, J. Yang ${ }^{2}$, L. Li ${ }^{2}$ and Y.C. He \\ ${ }^{1}$ Department of Anatomy, Harbin Medical University, Harbin, China \\ ${ }^{2}$ Department of Dermatology, Heilongjiang Provincial Hospital, Harbin, China \\ ${ }^{3}$ Department of Circulation, Heilongjiang Provincial Hospital, Harbin, China \\ ${ }^{4}$ Second Affiliated Hospital, Harbin Medical University, Harbin, China \\ *These authors contributed equally to this study. \\ Corresponding author: Y.C. He \\ E-mail: heyechun2010@163.com
}

Genet. Mol. Res. 11 (4): 3852-3860 (2012)

Received November 3, 2011

Accepted March 12, 2012

Published November 12, 2012

DOI http://dx.doi.org/10.4238/2012.November.12.2

\begin{abstract}
Association between the XRCC1 Arg399Gln polymorphism and susceptibility to gastric cancer has been investigated; overall, the results have been inconclusive. We made a meta-analysis of 13 case-control studies, including 3278 cases and 6243 controls. Crude odds ratios (OR) with $95 \%$ confidence intervals $(95 \% \mathrm{CI})$ were used to assess this possible association. We found no evidence of a significant association between the XRCC1 Arg399Gln polymorphism and gastric cancer risk (in the additive inheritance model, $\mathrm{OR}=0.986,95 \% \mathrm{CI}=$ $0.831-1.156$, in the dominant inheritance model, $\mathrm{OR}=1.044,95 \% \mathrm{CI}$ $=0.890-1.224$ and in the recessive inheritance model, $\mathrm{OR}=0.975$, $95 \% \mathrm{CI}=0.894-1.063$ ). We conclude that the XRCC1 Arg399Gln polymorphism is not a risk factor for developing gastric cancer.
\end{abstract}

Key words: XRCC1; Polymorphism; Gastric cancer; Susceptibility; Meta-analysis 


\section{INTRODUCTION}

Gastric cancer remains the second leading cause of cancer-related death and the fourth most common epithelial neoplasia worldwide (Bray et al., 2002; Parkin et al., 2005). Several environmental factors interact causing a cumulative effect in the early steps of gastric carcinogenesis, such as tobacco use (Lee and Hamling, 2009), dietary habit (Gonzalez and LopezCarrillo, 2010), and Helicobacter pylori infection (Malfertheiner et al., 2010). However, not all of those who are exposed to the risk factors will develop gastric cancer, suggesting interindividual differences in susceptibility.

DNA repair pathways are responsible for maintaining the integrity of the genome in the face of environmental insults and general DNA replication errors, playing a protective role against mutations that lead to cancer (Lindahl, 2000). Among DNA repair systems, the base excision repair (BER) pathway is responsible for the repair of oxidative DNA damage and single-strand breaks. The X-ray repair cross-complementation group 1 (XRCC1) protein plays an important role in BER (Hung et al., 2005; Li et al., 2009). The XRCC1 protein acts as a scaffolding protein for BER and single-strand break repair. The common polymorphism within the XRCC1 is Arg399Gln (rs25487), which is a G to A substitution at codon 399 in exon 10 of the gene, leading to the amino acid alteration arginine (Arg) to glutamine (Gln). The Arg399Gln polymorphism occurs at a conserved residue in the poly(ADP-ribose) polymerase binding domain of XRCC1. The $399 \mathrm{Gln}$ allele has been significantly associated with a higher level of DNA adducts, RBC glycophorin A mutations, micronuclei, sister chromatid exchanges, chromosomal damage, and prolonged cell cycle delay (Lei et al., 2002; Wang et al., 2003; Qu et al., 2005).

Shen et al. (2000) first reported an association between XRCC1 codon 399 polymorphisms and gastric cancer. Since then, several studies have reported the role of the XRCC1 Arg399Gln polymorphism in gastric cancer risk, but the results are inconclusive, partially because of the possibly pigmy effect of the polymorphism on gastric cancer risk and the relatively small sample size in each of the published studies. Recently, Chen et al. (2012) carried out a meta-analysis to test the association of the XRCC1 polymorphism with the risk of gastric cancer, but they did not exclude the studies which departure from Hardy-Weinberg equilibrium (HWE). Therefore, we repeated this meta-analysis to derive a more precise estimation of these associations.

\section{MATERIAL AND METHODS}

\section{Identification of studies}

To identify all studies that examined the association of XRCC1 Arg399Gln polymorphisms with gastric cancer, we performed a literature search in the Medline, EMBASE, OVID, ScienceDirect, and Chinese National Knowledge Infrastructure (CNKI) databases, without a language limitation, covering all papers published up to June 2011, using the following key words and subject terms: XRCC1, polymorphism, stomach neoplasms, and gastric cancer. We evaluated potentially relevant publications by checking their titles and abstracts and then obtained the most relevant publications for a detailed examination. Moreover, the reference lists of the selected papers were also screened for other potential articles that may have been 
missed in the initial search. Only published studies with full-text articles were included. When more than one of the same patient population was included in several publications, only the most recent or complete study was used in this meta-analysis.

\section{Selection criteria}

The following criteria were used for selection of reports for the meta-analysis: a) evaluation of the XRCC1 Arg399Gln polymorphism and gastric cancer risk, b) case-control studies, c) sufficient data to estimate an odds ratio (OR) and 95\% confidence interval (95\%CI), and d) genotype distribution of control population needed to be in HWE. After searching, we reviewed all papers in accordance with the criteria defined above for further analysis.

\section{Data extraction}

Data were carefully extracted from all eligible publications independently by two of the authors according to the inclusion criteria mentioned above. Disagreement was resolved by discussion between the two authors. If these two authors could not reach a consensus, another author was consulted to resolve the dispute and a final decision was reached based on the majority of the votes. The following data were collected from each study: first author's name, publication date, ethnicity, genotyping methods, genotype frequency, and the design of experiment for obtaining genotyping information on the XRCC1 Arg399Gln polymorphism. Different ethnicities were categorized as Caucasian, Asian and Latin American based on the place of recruitment of the subjects. We did not define any minimum number of patients to include in our meta-analysis.

\section{Statistical analysis}

Crude ORs with $95 \%$ CIs were used to assess the strength of association between the XRCC1 Arg399Gln polymorphism and gastric cancer risk. The pooled ORs were performed for an additive model (Arg/Arg versus Gln/Gln), a dominant model (Arg/Arg + Arg/ Gln versus Gln/Gln) and a recessive model (Arg/Arg versus Arg/Gln + Gln/Gln). The chisquare-based Q-statistical test was performed to assess heterogeneity among studies (Lau et al., 1997). P > 0.05 for the Q-test indicated a lack of heterogeneity among studies, so the pooled OR estimate of each study was calculated by the fixed-effects model [MantelHaenszel (1959) method]. Otherwise, the random-effects model [DerSimonian and Laird (1986) method] was used. Subgroup analyses were performed by ethnicity and study design. Sensitivity analysis was performed to assess the stability of the results. A single study involved in the meta-analysis was deleted each time to reflect the influence of the individual data set to the pooled ORs. An estimate of potential publication bias was assessed by visual inspection of funnel plots (Munafo et al., 2004), in which the standard error of $\log (\mathrm{OR})$ of each study was plotted against its $\log (\mathrm{OR})$. An asymmetric plot indicated a possible publication bias. The symmetry of the funnel plot was further evaluated by the Egger linear regression test $(\mathrm{P}<0.05$ was considered to be indicative of significant publication bias) (Egger et al., 1997). Statistical analysis was performed using STATA version 10.1 (Stata Corporation, College Station, TX, USA). 


\section{RESULTS}

\section{Study characteristics}

Through literature search and selection based on the inclusion criteria, 13 studies met our inclusion criteria (Shen et al., 2000; Lee et al., 2002; Ratnasinghe et al., 2004; Duarte et al., 2005; Huang et al., 2005; Miao et al., 2006; Song et al., 2006; Zhang et al., 2006; Ruzzo et al., 2007; Capella et al., 2008; Li et al., 2009; Yan et al., 2009; Palli et al., 2010) (Figure 1 and Table 1). The data for this analysis included 3278 cases and 6243 controls. Table 1 lists the identified studies and their main characteristics. There were 4 studies of Caucasians, 8 studies of Asians and one study of Latin Americans. Almost all of the cases were pathologically confirmed. Controls were mainly healthy populations and matched for age. Among these studies, 9 were population-based and two were hospital-based case control studies.

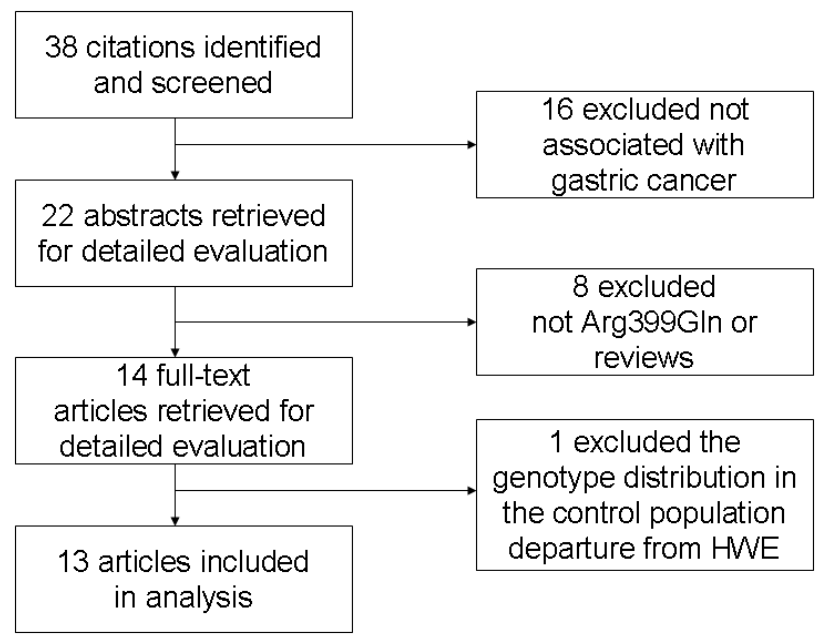

Figure 1. Flow diagram of included/excluded studies. HWE = Hardy-Weinberg equilibrium.

\begin{tabular}{|c|c|c|c|c|c|c|c|c|c|c|c|c|c|}
\hline \multirow[t]{2}{*}{ Author } & \multirow[t]{2}{*}{ Year } & \multirow[t]{2}{*}{ Ethnicity } & \multirow[t]{2}{*}{ Design } & \multirow[t]{2}{*}{ Method } & \multirow[t]{2}{*}{ Case } & \multirow[t]{2}{*}{ Control } & \multicolumn{3}{|c|}{ Case } & \multicolumn{3}{|c|}{ Control } & \multirow[t]{2}{*}{ HWE (P) } \\
\hline & & & & & & & GG & GA & $\overline{\mathrm{AA}}$ & $\overline{\mathrm{GG}}$ & GA & $\overline{\mathrm{AA}}$ & \\
\hline Capella' & 2008 & Caucasian & Nested & DHPLC & 245 & 1173 & 100 & 114 & 31 & 473 & 545 & 155 & 0.919 \\
\hline Duarte & 2005 & Latin American & PB & PCR-RFLP & 160 & 150 & 73 & 67 & 20 & 70 & 57 & 23 & 0.054 \\
\hline Huang & 2005 & Caucasian & PB & MALDI-TOF MS & 281 & 390 & 124 & 121 & 36 & 166 & 179 & 45 & 0.756 \\
\hline Lee & 2002 & Asian & HB & PCR-RFLP & 190 & 172 & 110 & 71 & 9 & 94 & 69 & 9 & 0.419 \\
\hline Miao & 2006 & Asian & PB & PCR-RFLP & 500 & 1000 & 221 & 234 & 45 & 532 & 402 & 66 & 0.392 \\
\hline Ratnasinghe & 2004 & Asian & Cohort & Taqman & 86 & 418 & 49 & 34 & 3 & 192 & 193 & 33 & 0.103 \\
\hline Shen & 2000 & Asian & PB & PCR-RFLP & 188 & 166 & 92 & 83 & 13 & 94 & 59 & 13 & 0.387 \\
\hline Song & 2006 & Asian & PB & DHPLC & 102 & 101 & 46 & 48 & 8 & 54 & 44 & 3 & 0.088 \\
\hline Yan & 2009 & Asian & PB & PCR-RFLP & 455 & 650 & 241 & 191 & 23 & 345 & 270 & 35 & 0.054 \\
\hline Zhang & 2006 & Asian & PB & PCR-RFLP & 236 & 708 & 136 & 82 & 18 & 369 & 275 & 64 & 0.219 \\
\hline Ruzzo & 2007 & Caucasian & PB & PCR-RFLP & 91 & 119 & 36 & 45 & 10 & 50 & 59 & 10 & 0.198 \\
\hline $\mathrm{Li}$ & 2009 & Asian & $\mathrm{HB}$ & PCR-RFLP & 455 & 650 & 241 & 191 & 23 & 345 & 270 & 35 & 0.055 \\
\hline Palli & 2010 & Caucasian & PB & Taqman & 289 & 546 & 123 & 137 & 29 & 250 & 233 & 63 & 0.439 \\
\hline
\end{tabular}

$\mathrm{HB}=$ hospital-based study; $\mathrm{PB}=$ population-based study; $\mathrm{HWE}=$ Hardy-Weinberg equilibrium. 


\section{Meta-analysis results}

Table 2 lists the main results of the meta-analysis. The overall data showed that the individuals who carried the Arg/Arg genotype did not significantly increase gastric cancer risk compared to those carrying the $\mathrm{Gln} / \mathrm{Gln}$ genotype (additive model, $\mathrm{OR}=0.986,95 \% \mathrm{CI}=0.831$ 1.156) (Figure 2), and no significant association was found in the dominant model (OR $=1.044$, $95 \% \mathrm{CI}=0.890-1.224)$ or recessive model $(\mathrm{OR}=0.975,95 \% \mathrm{CI}=0.894-1.063)$. Thus, the 13 studies were analyzed by stratification based on ethnicity and study design. In the subgroup analysis of ethnicity, there was no significant association between the polymorphism and gastric cancer in Caucasians and Asians. When stratified by study design, there was no significant association between the polymorphism and gastric cancer risk; the details are listed in Table 2 .

\begin{tabular}{|c|c|c|c|c|c|c|c|}
\hline \multirow[t]{2}{*}{ Subgroup } & \multirow[t]{2}{*}{ Number of comparisons } & \multicolumn{2}{|c|}{ GG $v s$ AA } & \multicolumn{2}{|c|}{$(\mathrm{GG}+\mathrm{GA}) v s \mathrm{AA}$} & \multicolumn{2}{|c|}{$\mathrm{GG} v s(\mathrm{GA}+\mathrm{AA})$} \\
\hline & & OR $(95 \% \mathrm{CI})$ & I-squared (\%) & OR $(95 \% \mathrm{CI})$ & I-squared (\%) & OR $(95 \% \mathrm{CI})$ & I-squared (\%) \\
\hline \multicolumn{8}{|l|}{ Ethnicity } \\
\hline Caucasian & 4 & $0.99(0.77-1.30)$ & 0.0 & $1.01(0.79-1.29)$ & 0.0 & $0.98(0.83-1.14)$ & 0.0 \\
\hline Asian & 8 & $0.95(0.76-1.18)$ & 40.0 & $1.05(0.84-1.31)$ & 35.2 & $0.98(0.88-1.08)$ & 68.2 \\
\hline \multicolumn{8}{|l|}{ Design } \\
\hline $\mathrm{PB}$ & 9 & $0.93(0.77-1.12)$ & 4.2 & $1.02(0.85-1.21)$ & 13.8 & $0.94(0.86-1.04)$ & 50.2 \\
\hline Total & 13 & $0.98(0.83-1.16)$ & 6.6 & $1.04(0.89-1.22)$ & 2.9 & $0.98(0.89-1.06)$ & 47.9 \\
\hline
\end{tabular}

$\mathrm{PB}=$ population-based study; I-squared $=$ the variation in $\mathrm{OR}$ attributable to heterogeneity. $95 \% \mathrm{CI}=95 \%$ confidence interval.

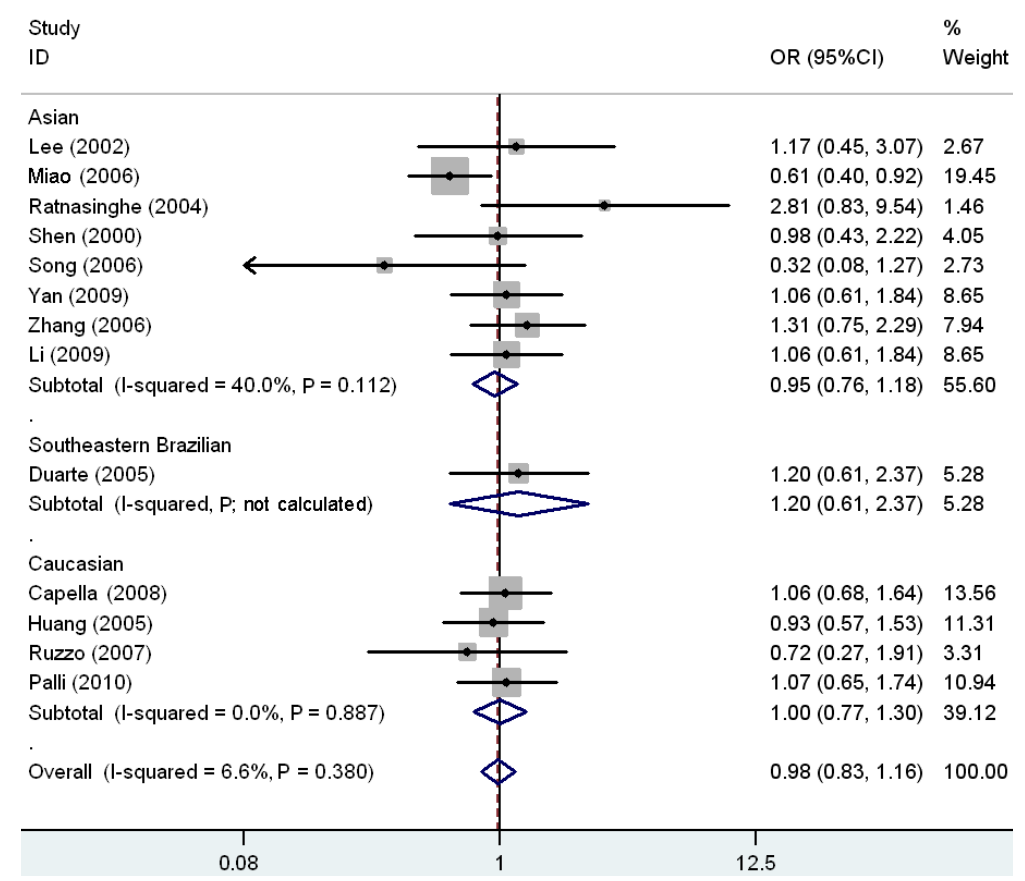

Figure 2. Forest plot of gastric cancer risk associated with the GG genotype compared to the AA genotype. OR = odds ratio; $95 \% \mathrm{CI}=95 \%$ confidence interval. 


\section{Sensitivity analysis}

Sensitivity analyses were conducted to determine whether modification of the inclusion criteria of the meta-analysis affected the final results. A single study involved in the meta-analysis was deleted each time to reflect the influence of the individual dataset to the pooled ORs, and the corresponding pooled ORs were not materially altered (data not shown), indicating that our results were statistically robust.

\section{Publication bias}

Begg's funnel plots and the Egger test were used to assess publication bias. The shapes of the funnel plots revealed no obvious asymmetry (Figure 3). The Egger test was then used to statistically assess funnel plot symmetry. The results suggested no evidence of publication bias $(\mathrm{P}=0.849$ for additive model, $\mathrm{P}=0.955$ for dominant model and $\mathrm{P}=0.811$ for recessive model). These findings indicated that the results of these meta-analyses were relatively stable and that publication bias unlikely affected the results of the meta-analyses.

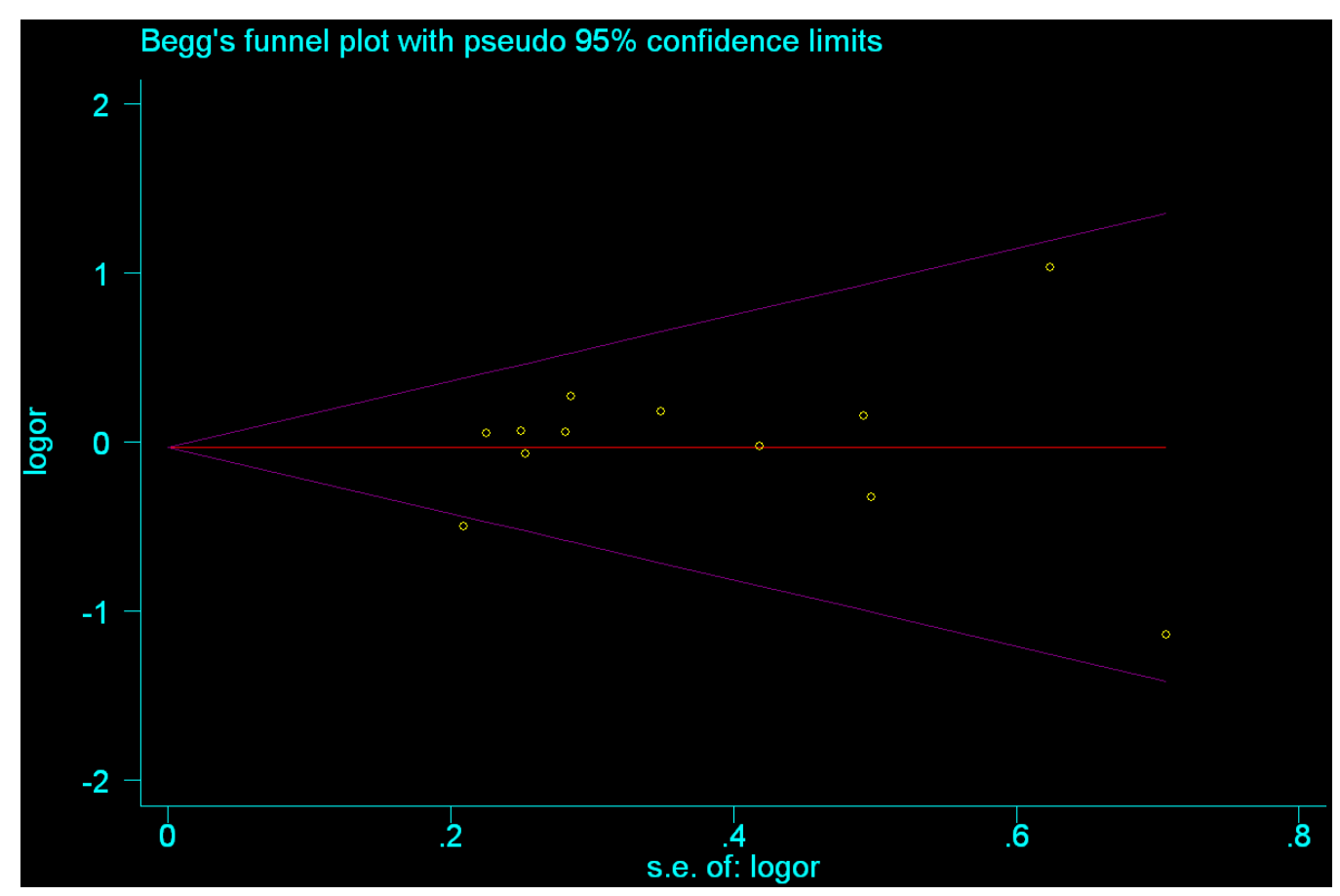

Figure 3. Begg's funnel plot of XRCC1 Arg399Gln and gastric cancer risk (GG vs AA).

\section{DISCUSSION}

The XRCC1 protein is an important component of the BER pathway, which fixes base damage and DNA single-strand breaks caused by ionizing radiation and alkylating agents (Marintchev 
et al., 1999). The Arg399Gln polymorphism is located in the region of the BRCT-I interaction domain of XRCC1 with ADP-ribose polymerase. The presence of the variant 399Gln allele has been shown to be associated with measurably reduced DNA repair capacity as assessed by the persistence of DNA adducts, tumor-suppressor gene P53 mutations, increased red blood cell glycophorin A, elevated levels of sister chromatid exchanges, and prolonged cell-cycle delay (Lunn et al., 1999).

For the relatively small sample size in each of the published studies, it is important to accumulate data from different studies to provide evidence for the association of XRCC1 Arg399Gln with gastric cancer risk. There are some meta-analyses that have tested the associations between XRCC1 Arg399Gln and gastric cancer risk, but they had some limitations. Either the inclusion studies were small (Geng et al., 2008) or the analysis included studies with departure from HWE (Chen et al., 2012). Therefore, we repeated the analysis, and in this meta-analysis, we included a total of 3278 cases and 6243 controls from 13 studies to investigate the associations of the XRCC1 Arg399Gln polymorphism with gastric cancer risk. We found that there were no significant associations between the XRCC1 Arg399Gln polymorphism and gastric cancer risk. In the subgroup analysis of ethnicity and study design, there was also no association.

The association between the XRCC1 Arg399Gln polymorphism and the risk of different kinds of cancers has been extensively studied. Previous meta-analyses indicated that the XRCC1 Arg399Gln polymorphism is not significantly associated with colorectal cancer risk (Wang et al., 2010) or bladder cancer (Wang et al., 2008). However, this polymorphism is associated with the risk of lung cancer (Kiyohara et al., 2006), breast cancer (Saadat and Ansari-Lari, 2009) and prostate cancer (Geng et al., 2009). Thus, the role of this polymorphism in the risk of cancers varies. In the present study, we found that the XRCC1 Arg399Gln polymorphism is not a risk factor for gastric cancer. The effects of this polymorphism on susceptibility to cancer may differ according to cancer type. It may not be uncommon for the same polymorphism to play different roles in cancer susceptibility across different tumor locations, because cancer is an extremely complex disease and because genetic heterogeneity exists in different cancer types (Hirschhorn et al., 2002). Different kinds of cancer should have a different genetic susceptibility.

There are some limitations to this meta-analysis. First, only published studies were included in the meta-analysis. It is possible that some related unpublished studies that might have met the inclusion criteria were missed; therefore, publication bias may have been present, even though statistical analysis indicated this not to be the case. Second, our results were based on unadjusted estimates, and a more precise analysis could have been conducted if individual data were available; this would allow adjustment by other covariates such as age, ethnicity, environmental factors, and lifestyle. Third, in the subgroup analyses, the number of Caucasians was relatively small, not having enough statistical power to explore the association of the polymorphism with gastric cancer susceptibility. However, our meta-analysis also had some advantages. First, substantial numbers of cases and controls were pooled from different studies, which significantly increased the statistical power of the analysis. Second, no publication bias was detected, indicating that the pooled result should be reliable.

In summary, our meta-analysis indicates that genetic variations of the XRCC1 Arg399Gln do not have an association with gastric cancer risk.

\section{ACKNOWLEDGMENTS}

Research supported by the International Science and Technology Cooperation Funds of Heilongjiang Province, China (\#2006W0089-00). 


\section{REFERENCE}

Bray F, Sankila R, Ferlay J and Parkin DM (2002). Estimates of cancer incidence and mortality in Europe in 1995. Eur. J. Cancer 38: 99-166.

Capella G, Pera G, Sala N, Agudo A, et al. (2008). DNA repair polymorphisms and the risk of stomach adenocarcinoma and severe chronic gastritis in the EPIC-EURGAST study. Int. J. Epidemiol. 37: 1316-1325.

Chen B, Zhou Y, Yang P and Wu XT (2012). Polymorphisms of XRCC1 and gastric cancer susceptibility: a meta-analysis. Mol. Biol. Rep. 39: 1305-1313.

DerSimonian R and Laird N (1986). Meta-analysis in clinical trials. Control. Clin. Trials 7: 177-188.

Duarte MC, Colombo J, Rossit AR, Caetano A, et al. (2005). Polymorphisms of DNA repair genes XRCC1 and XRCC3, interaction with environmental exposure and risk of chronic gastritis and gastric cancer. World J. Gastroenterol. 11: 6593-6600.

Egger M, Davey SG, Schneider M and Minder C (1997). Bias in meta-analysis detected by a simple, graphical test. BMJ 315: 629-634.

Geng J, Zhang YW, Huang GC and Chen LB (2008). XRCC1 genetic polymorphism Arg399Gln and gastric cancer risk: A meta-analysis. World J. Gastroenterol. 14: 6733-6737.

Geng J, Zhang Q, Zhu C, Wang J, et al. (2009). XRCC1 genetic polymorphism Arg399Gln and prostate cancer risk: a meta-analysis. Urology 74: 648-653.

Gonzalez CA and Lopez-Carrillo L (2010). Helicobacter pylori, nutrition and smoking interactions: their impact in gastric carcinogenesis. Scand. J. Gastroenterol. 45: 6-14.

Hirschhorn JN, Lohmueller K, Byrne E and Hirschhorn K (2002). A comprehensive review of genetic association studies. Genet. Med. 4: 45-61.

Huang WY, Chow WH, Rothman N, Lissowska J, et al. (2005). Selected DNA repair polymorphisms and gastric cancer in Poland. Carcinogenesis 26: 1354-1359.

Hung RJ, Hall J, Brennan P and Boffetta P (2005). Genetic polymorphisms in the base excision repair pathway and cancer risk: a HuGE review. Am. J. Epidemiol. 162: 925-942.

Kiyohara C, Takayama K and Nakanishi Y (2006). Association of genetic polymorphisms in the base excision repair pathway with lung cancer risk: a meta-analysis. Lung. Cancer 54: 267-283.

Lau J, Ioannidis JP and Schmid CH (1997). Quantitative synthesis in systematic reviews. Ann. Intern. Med. 127: 820-826. Lee PN and Hamling J (2009). The relation between smokeless tobacco and cancer in Northern Europe and North America. A commentary on differences between the conclusions reached by two recent reviews. BMC Cancer 9: 256 .

Lee SG, Kim B, Choi J, Kim C, et al. (2002). Genetic polymorphisms of XRCC1 and risk of gastric cancer. Cancer Lett. 187: 53-60.

Lei YC, Hwang SJ, Chang CC, Kuo HW, et al. (2002). Effects on sister chromatid exchange frequency of polymorphisms in DNA repair gene XRCC1 in smokers. Mutat. Res. 519: 93-101.

Li WQ, Zhang L, Ma JL, Zhang Y, et al. (2009). Association between genetic polymorphisms of DNA base excision repair genes and evolution of precancerous gastric lesions in a Chinese population. Carcinogenesis 30: 500-505.

Lindahl T (2000). Suppression of spontaneous mutagenesis in human cells by DNA base excision-repair. Mutat. Res. 462: 129-135.

Lunn RM, Langlois RG, Hsieh LL, Thompson CL, et al. (1999). XRCC1 polymorphisms: effects on aflatoxin B1-DNA adducts and glycophorin A variant frequency. Cancer Res. 59: 2557-2561.

Malfertheiner P, Bornschein J and Selgrad M (2010). Role of Helicobacter pylori infection in gastric cancer pathogenesis: a chance for prevention. J. Dig. Dis. 11: 2-11.

Mantel N and Haenszel W (1959). Statistical aspects of the analysis of data from retrospective studies of disease. J. Natl. Cancer Inst. 22: 719-748.

Marintchev A, Mullen MA, Maciejewski MW, Pan B, et al. (1999). Solution structure of the single-strand break repair protein XRCC1 N-terminal domain. Nat. Struct. Biol. 6: 884-893.

Miao X, Zhang X, Zhang L, Guo Y, et al. (2006). Adenosine diphosphate ribosyl transferase and x-ray repair crosscomplementing 1 polymorphisms in gastric cardia cancer. Gastroenterology 131: 420-427.

Munafo MR, Clark TG and Flint J (2004). Assessing publication bias in genetic association studies: evidence from a recent meta-analysis. Psychiatry Res. 129: 39-44.

Palli D, Polidoro S, D’Errico M, Saieva C, et al. (2010). Polymorphic DNA repair and metabolic genes: a multigenic study on gastric cancer. Mutagenesis 25: 569-575.

Parkin DM, Bray F, Ferlay J and Pisani P (2005). Global cancer statistics, 2002. CA Cancer J. Clin. 55: 74-108.

Qu T, Morii E, Oboki K, Lu Y, et al. (2005). Micronuclei in EM9 cells expressing polymorphic forms of human XRCC1. Cancer Lett. 221: 91-95. 
Ratnasinghe LD, Abnet C, Qiao YL, Modali R, et al. (2004). Polymorphisms of XRCC1 and risk of esophageal and gastric cardia cancer. Cancer Lett. 216: 157-164.

Ruzzo A, Canestrari E, Maltese P, Pizzagalli F, et al. (2007). Polymorphisms in genes involved in DNA repair and metabolism of xenobiotics in individual susceptibility to sporadic diffuse gastric cancer. Clin. Chem. Lab. Med. 45: 822-828.

Saadat M and Ansari-Lari M (2009). Polymorphism of XRCC1 (at codon 399) and susceptibility to breast cancer, a metaanalysis of the literatures. Breast Cancer Res. Treat. 115: 137-144.

Shen H, Xu Y, Qian Y, Yu R, et al. (2000). Polymorphisms of the DNA repair gene XRCC1 and risk of gastric cancer in a Chinese population. Int. J. Cancer 88: 601-606.

Song CG, Lu HS, Huang CM, Liu X, et al. (2006). Relationship between gene polymorphism of XRCC1 Arg399Gln and the risk of gastric cancer patients in Fujian. Zhonghua Shiyan Waike Zazhi. 23: 1021.

Wang B, Wang D, Huang G, Zhang C, et al. (2010). XRCC1 polymorphisms and risk of colorectal cancer: a meta-analysis. Int. J. Colorectal Dis. 25: 313-321.

Wang C, Sun Y and Han R (2008). XRCC1 genetic polymorphisms and bladder cancer susceptibility: a meta-analysis. Urology 72: 869-872.

Wang Y, Spitz MR, Zhu Y, Dong Q, et al. (2003). From genotype to phenotype: correlating XRCC1 polymorphisms with mutagen sensitivity. DNA Repair 2: 901-908.

Yan L, Yanan D, Donglan S, Na W, et al. (2009). Polymorphisms of XRCC1 gene and risk of gastric cardiac adenocarcinoma. Dis. Esophagus 22: 396-401.

Zhang Z, Miao XP, Tan W, Guo YL, et al. (2006). Correlation of genetic polymorphisms in DNA repair genes ADPRT and XRCC1 to risk of gastric cancer. Ai Zheng 25: 7-10. 